\title{
Investigation into the high transients experienced in Eleonore Mine's pastefill distribution system
}

\author{
Maureen Mcguinness \& Kelvin Creber \\ Paterson \& Cooke Canada Inc., Sudbury, Canada \\ Justin Jacobs \\ Paterson \& Cooke USA, Denver, USA \\ Bernard Haley \\ Newmont Goldcorp, Rouyn-Noranda, Canada
}

SUMMARY: Eleonore Mine experiences high transient forces in their pastefill underground distribution system which results in significant damage to the pipeline supports. This investigation examines the causes of the transients in this pumped system and looks at their effect.

A transient flow model was developed and validated using operating data. The investigation showed that favourable transient conditions came from operation in full flow conditions. It was also shown that a misaligned hydraulic pulsation dampener can produce higher transients in the line than would be experienced without one. This investigation spurred design and operating strategy changes to combat transients in the underground distribution system.

Keywords: transients, positive displacement pump, backfill, piping

\section{INTRODUCTION}

An ongoing issue at Eleonore Mine has been high transient forces in the pastefill underground distribution system (UDS). These transients cause loud hammering underground with forces strong enough to cause damage to the pipeline supports. Pastefill production delays are common due to unexpected maintenance that is required to fix the UDS supports.

The goal of this investigation was to reduce the transients in the Eleonore UDS. In parallel to this work, improvements to alignment between the hydraulic pulsation dampener (HPD) and the positive displacement (PD) pump were on-going. It was acknowledged that there will always be times that the HPD is not operating at its optimal alignment. It was decided that the UDS should be upgraded to meet the maximum transient loads since they have resulted in damage to the system under the present conditions.

\section{BACKGROUND}

Transient forces in a pipeline are caused by fast moving pressure waves which in turn are a direct result of changes in flow rate. The mechanics of operation of a positive displacement piston pump inherently produces variation in flow rate. At every stroke change over, there is a short period of time in which there is no paste being pushed through the line. This sudden loss of flow causes a transient pressure wave to travel downstream in the pipeline. A HPD is 
added to the system to counter the loss in flow by slowly accumulating paste during a pump stroke and then pushing paste into the pipeline during the switch between the pump cylinders.

This investigation sought to understand the causes and magnitude of the transients in the system, to develop a strategy for their mitigation, and - since the transient forces cannot be fully removed - to review anchor standard design loads. The process taken was to capture insitu measurements of the transients in the system, and then model them - validating the data as appropriate. The model was then used to evaluate the effect that changes to the operating process has on the generated transient forces.

\subsection{Eleonore pastefill distribution system}

The pastefill distribution system at Eleonore is characterised by a short surface borehole after which the pipeline follows the ramp to 140 Level. Internal boreholes below 140 Level distribute paste to both the north and south side of the orebody as shown in Figure 1. The transient forces are reported to be more frequent and stronger in the upper mine, particularly above Level 140.

\subsection{Hydraulic modeling}

A typical hydraulic grade line (HGL) plot for a pour to the middle of the mine is shown in Figure 2. The distance between the HGL and the pipeline profile is an indication of the pressure in the pipeline at that point. This HGL plot indicates that the system requires a pump to

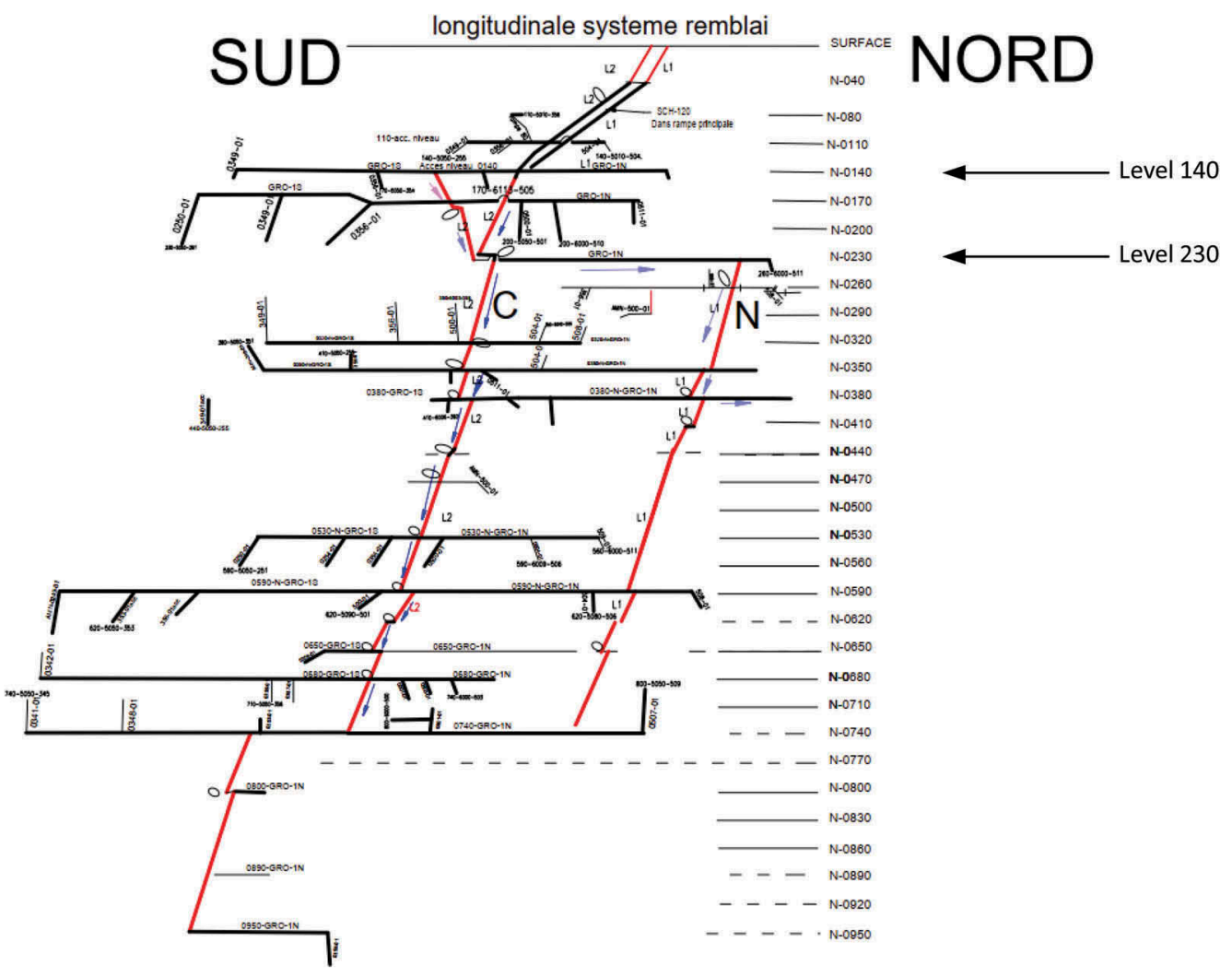

Figure 1. Eleonore Mine long section.

Source: Eleonore Mine 2019. 


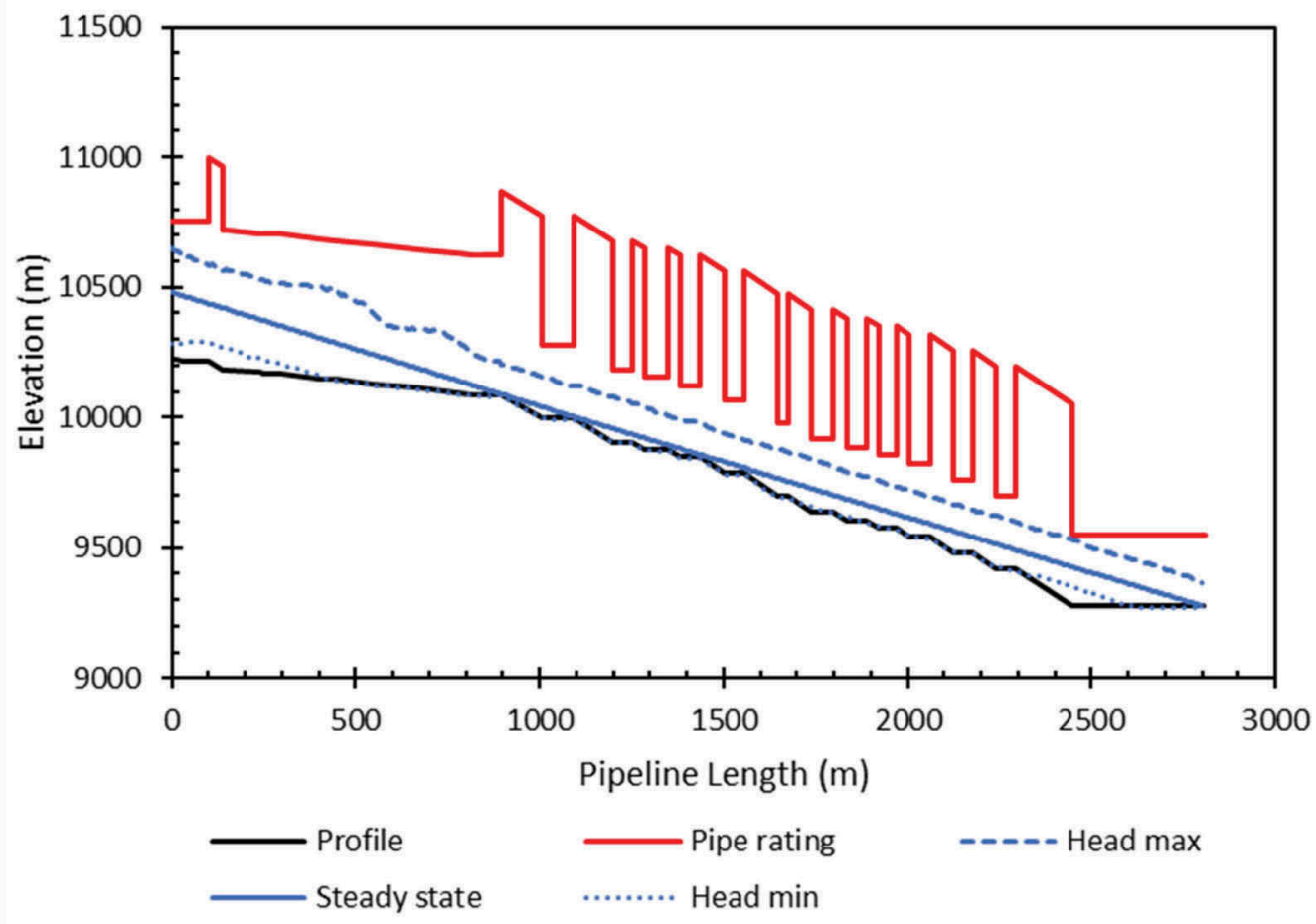

Figure 2. Example of Eleonore hydraulic grade line plot.

Source: Study.

transport the paste the stope location ${ }^{1}$. The interception of the grade line and the pipeline profile indicates an area in which slack flow exists; in this case at the end of the 140 level.

The maximum transient pressures are shown on the HGL plot as a dotted line above the HGL. The minimum pressure transient is shown below the HGL. These can add to the impact loading, as the pipeline is temporarily placed in slack flow.

\subsection{Positive displacement pump and HPD}

Details of the Eleonore Mine paste pump and HPD are outlined in Table 1. The Putzmeister KOS line of positive displacement pumps use an S-tube to switch between delivery cylinders. The HPD accumulates paste during the cylinder stroke and then discharges it into the pipeline during the short period of time that the S-tube switches to the other cylinder. This cycle is depicted in Figure 3, where the blue dots indicate paste discharged from cylinder \#1 and red dots from cylinder \#2.

\subsection{UDS supports}

The pipeline is supported with a series of hangers, guides and anchors. The anchors resist forces acting in the axial direction of the pipeline, which includes the transient forces. An

1. For a system to flow by gravity only, the hydraulic grade line must intercept the y-axis. The distance above the pipeline profile that the hydraulic grade line intercepts the $y$-axis provides the pumping requirements to ensure flow. 
Table 1. Eleonore positive displacement pump data.

\begin{tabular}{ll}
\hline Vendor & Putzmeister \\
\hline Model & KOS 25100 HP \\
Delivery cylinder diameter & $360 \mathrm{~mm}$ \\
Delivery cylinder length & $2500 \mathrm{~mm}$ \\
Max delivery pressure & $75 \mathrm{bar}$ \\
Dampener Model & HPD (High Pressure Dampener) \\
Dampener cylinder diameter & $279.4 \mathrm{~mm}$ \\
Dampener cylinder length & $1500 \mathrm{~mm}$ \\
\hline
\end{tabular}

Source: Eleonore Mine 2019.
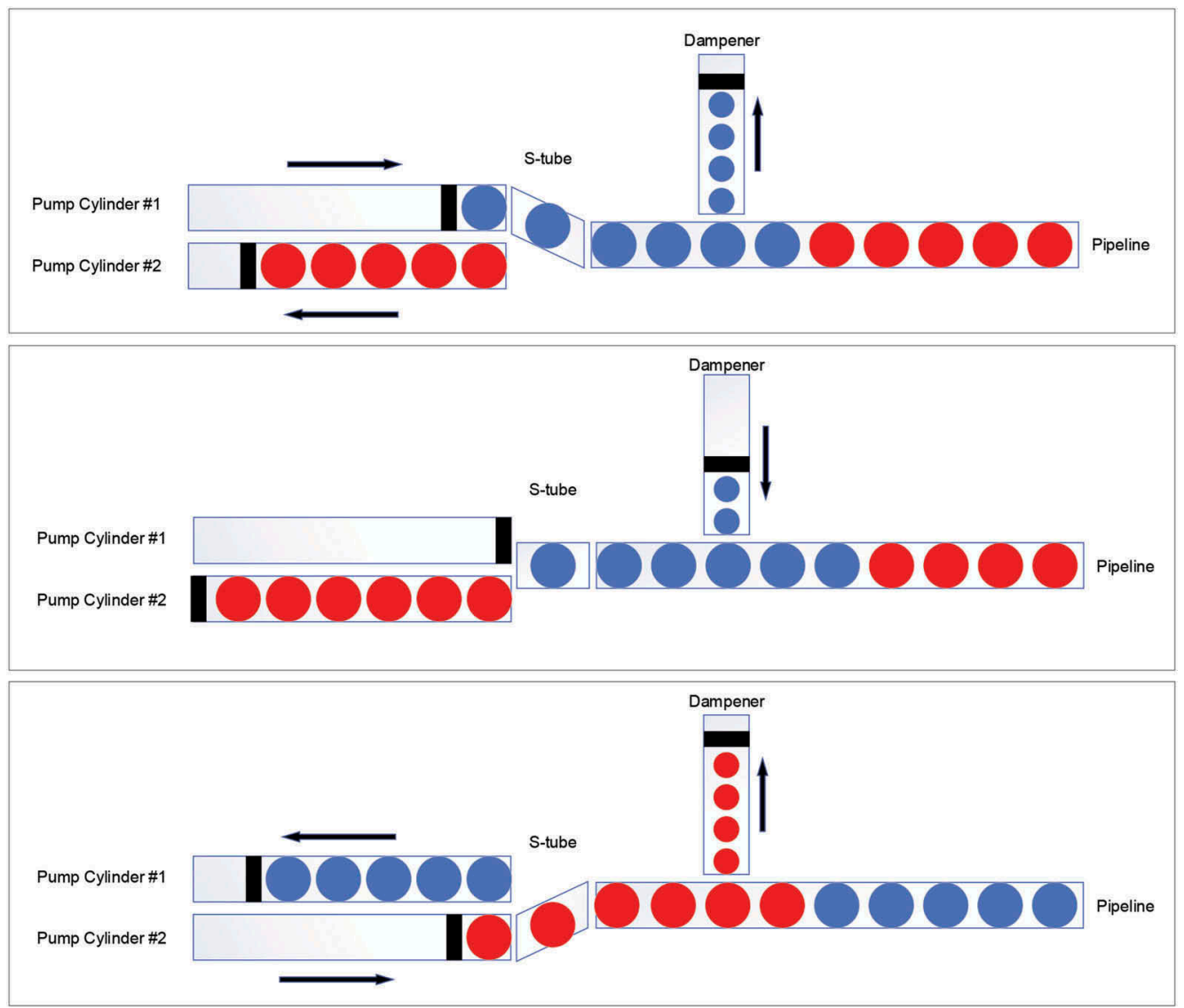

Figure 3. Cycle of paste flow during switch between PD pump cylinders and HPD.

Source: Based on schematic by Putzmeister.

example of an anchor used at Eleonore is provided in Figure 4. Two types of anchors are used: normal and heavy duty. The normal anchors were designed for $35 \mathrm{kN}$ under the design assumption that an HPD would be used with the PD pump operation and that this would reduce the transient forces. The heavy duty anchor was designed at $150 \mathrm{kN}$. This was intended to be installed in the upper mine for the operation when the HPD was not yet installed. 

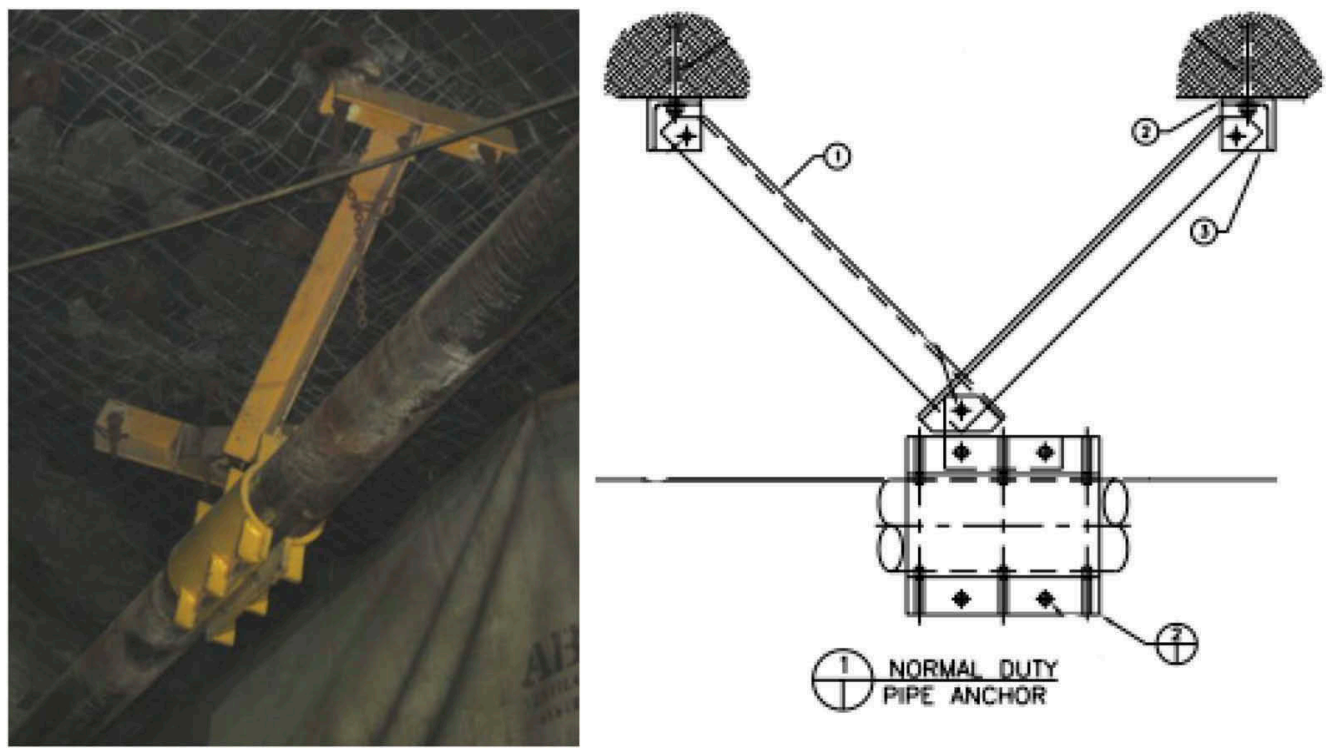

Figure 4. Example of anchor support used at Eleonore.

Source: Study.

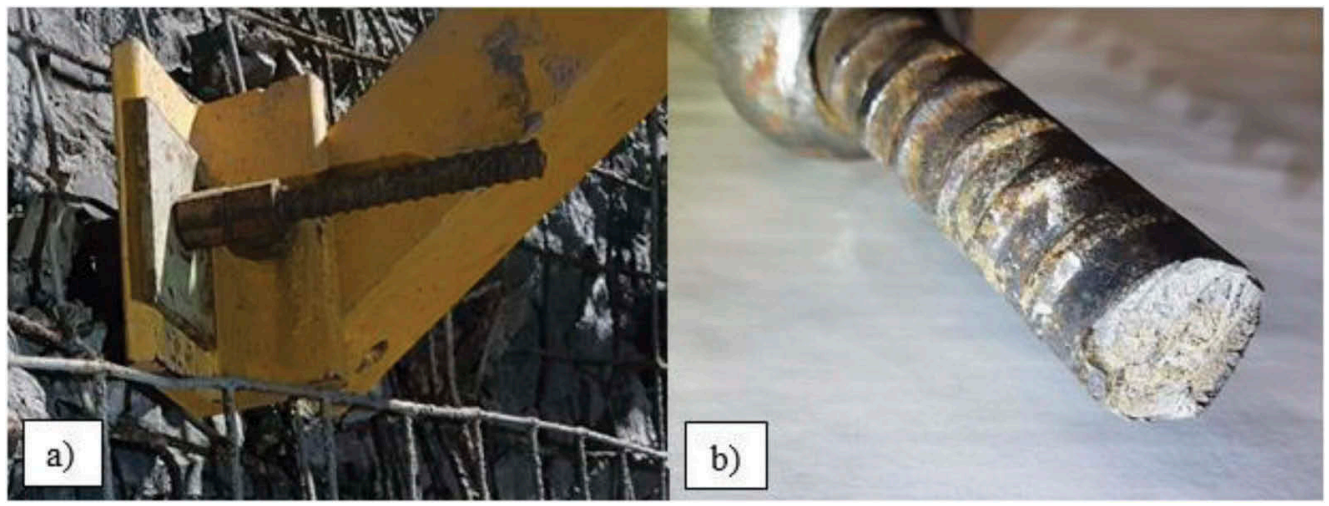

Figure 5. A) Gap behind footing, b) Rock bolt sheared by transient forces.

Source: Study

Proper installation of the anchors and guides in the system is paramount to maintaining the integrity of the UDS system. In particular, the inclusion of grouting behind the anchor and guide foot plates was shown to have a ten-fold effect on the strength of the support. Without grout, the bolts which have a gap between the footplate and the wall (Figure 5a) are placed in a cantilever situation with the bolt not able to be put under tension. The result is that this bolt becomes the weak point in the system and can break prematurely due to impact loading and fatigue. An example of a broken bolt is shown in Figure 5b.

\section{TRANSIENT MONITORING}

To understand the behaviour and magnitude of the transients that were passing through the Eleonore UDS, they first needed to be recorded. Two high speed pressure gauges were 


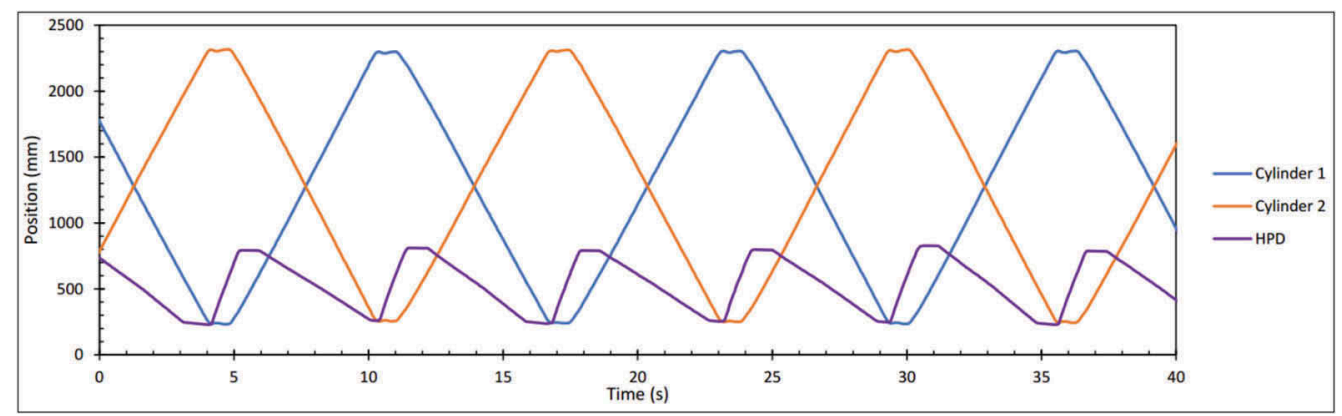

Figure 6. Example cylinder position graph.

Source: Study.

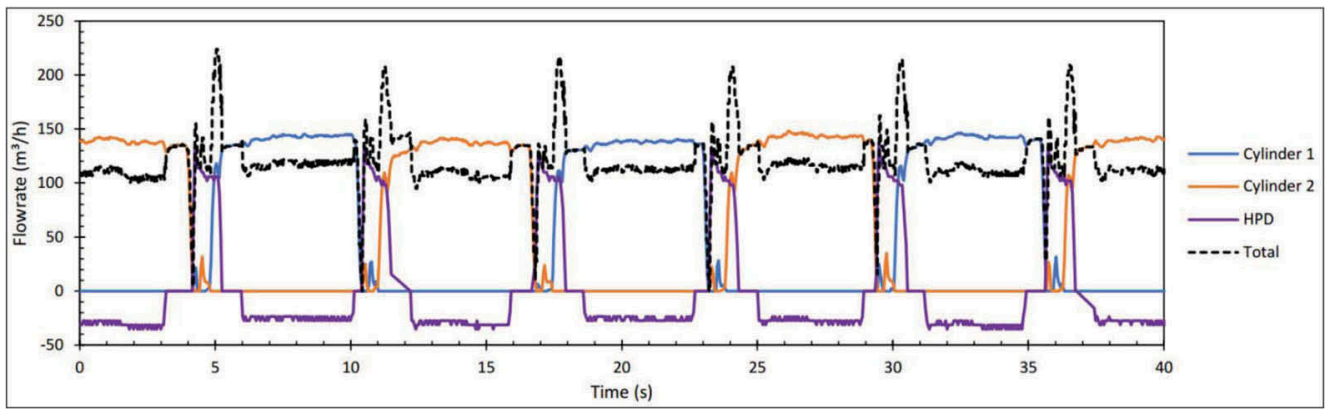

Figure 7. Example flow profile of the paste.

Source: Study.

installed on level 140, which was one of the areas experiencing the most damage. This was at the bottom of the ramp from surface. Two gauges, with a known distance apart and elevation, were installed. There was also a regular, low speed pressure gauge in this area. Comparing the pressures recorded from the regular gauge to the high speed gauges, it was shown that the regular gauges were picking up the general trend of the reoccurring peaks in pressure but the magnitude of these peaks was reduced.

Since transients are caused by changes in flow rate, the behaviour of the flow in the pipe was evaluated using the recorded position of the PD pump cylinders. Given the known cylinder geometry, a flowrate over time could be determined. Two position curves are traced at any time; one for each cylinder of the pump. The cylinders are connected hydraulically and therefore the position graph of one cylinder is a mirror image of the other as shown in Figure 6. The HPD position was also recorded. It is shown to move forward only during the time when both pistons are at zero flow.

The flow profile in the pipeline can be developed by combining the flow contributions of the two cylinders and the HPD as shown in Figure 7. The negative flow in the HPD indicates the time in which it was drawing paste into the cylinder from the pipeline. The effect does not occur for the PD pump cylinders because those cylinders draw the paste from the hopper before it enters the pipeline.

\section{TRANSIENT MODELLING}

Each of the three cylinder flowrates are inputted into the transient model. The paste properties (yield stress, viscosity, \% solids), pipe diameter and pipeline profile are also included in the 
model. The result of the modelling was a pressure profile over time at any point in the pipeline such as the one for level 140 pressure gauge location. This pressure profile was compared to the pressures measured in the field as shown in Figure 8.

The model was shown to have a similar result to the actual measurements. The amplitude of the pressure peaks aligned as did the frequency. This process was performed on three different occasions with similar results. From this validation, it was concluded that the model was representative of the Eleonore UDS system transients. This made the next steps of the study possible - to evaluate forces on anchors, and to evaluate the effect of operational changes on the transients experienced in the pipeline.

The transient pressures throughout the pipeline are then used to calculate axial force. A dynamic load factor (DLF) must be applied to all calculated loads to account for the amplification effect of the forces in dynamic conditions (ASME 2016). Based on past experience, and the natural frequencies of underground piping systems, a DLF of 1.75 was applied.

This procedure was followed for several pours spanning the range of the distribution system, including pours to the upper mine and lower mine both close and far from the main pipeline system. The maximum axial forces experienced for each pour are summarized in Figure 9.

Some key observations include that when the hydraulics are such that the 140 to 230 level borehole is in slack flow the transients are isolated in the upper portion of the pipeline, and that the transients in the upper mine sometimes exceed the $150 \mathrm{kN}$ load rating of the heavy duty anchors in the system. All cases are shown to exceed the $35 \mathrm{kN}$ load rating of the normal anchor.

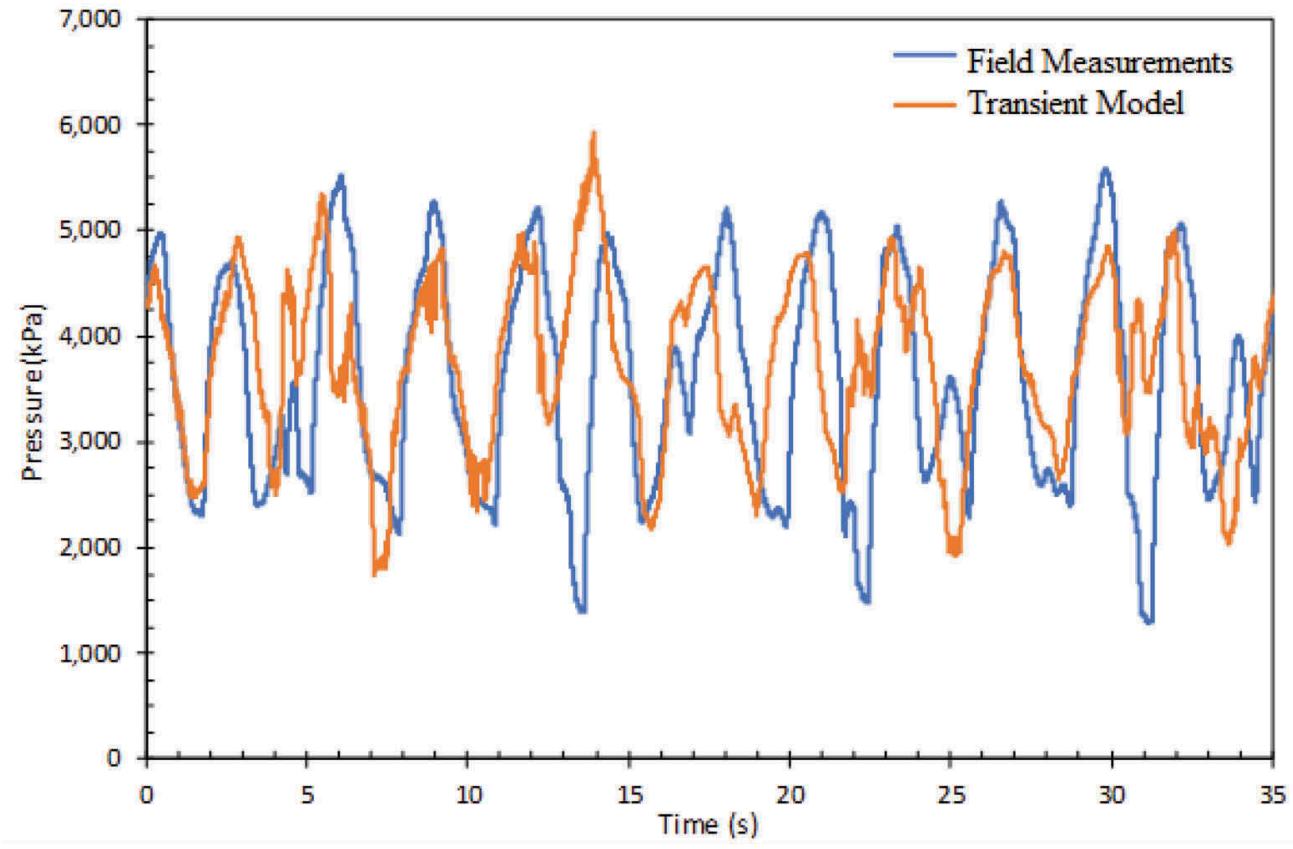

Figure 8. Transient model pressure predictions compared to actual measurements.

Source: Study. 


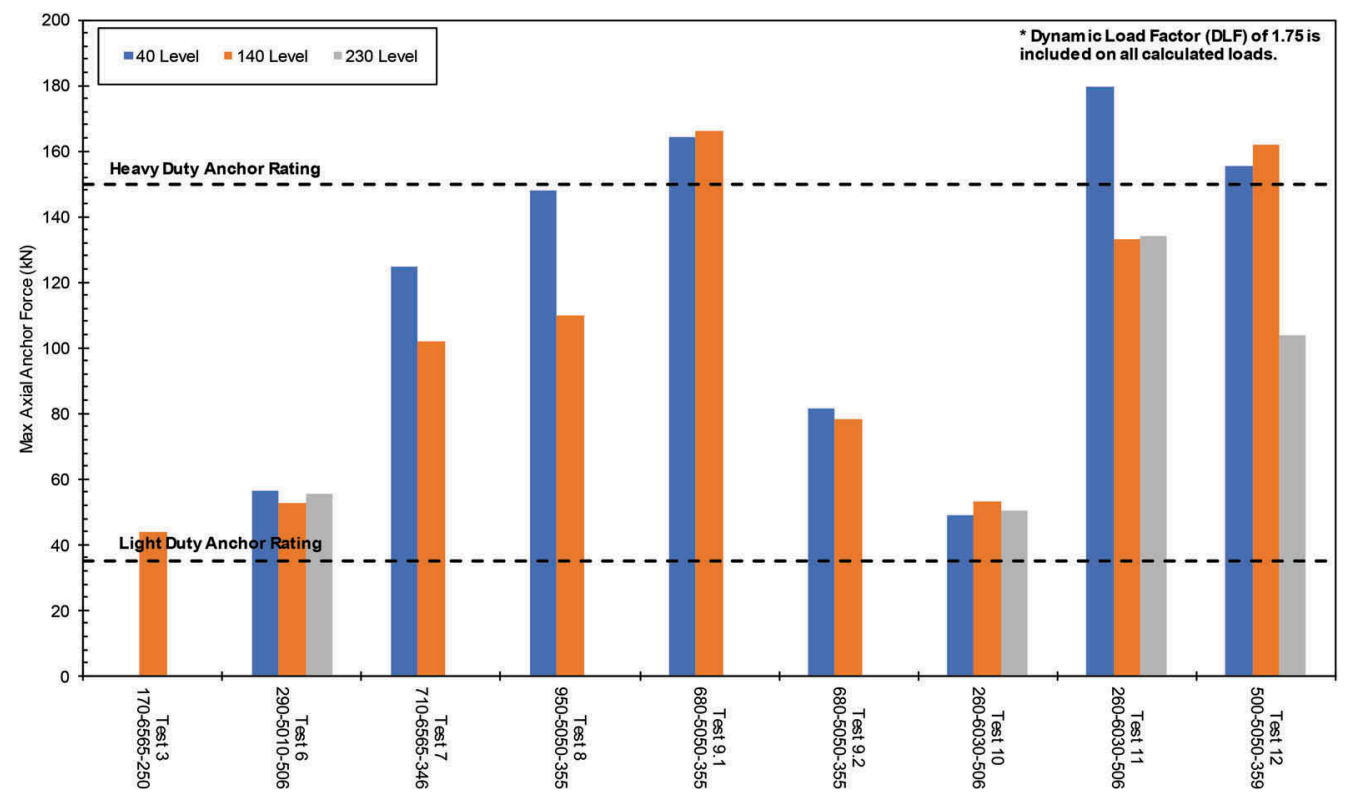

Figure 9. Summary of modeling results of the axial forces at levels 40, 140 and 230.

Source: Study.

\section{PROCESS PARAMETER EFFECT ON TRANSIENTS}

Using the validated transient model, investigations were made to assess the impact of solids concentration, the cylinder/HPD flowrates and timing.

\subsection{Effect of solids concentration on transients}

The operating practice to date was to maintain a low mass concentration as it was the operational experience that the transient forces were larger when the solids concentration increased. This was in contradiction to the authors' experience where increasing the concentration of the paste usually results in lower transients in the system. To examine this effect, the cylinder position and operating parameters were measured for a series of paste concentrations, visualized in Figure 10. The model confirmed that the higher concentrations resulted in the lowest transient forces in the pipeline. However, the site experience was also confirmed by the modelling as it was shown that the transient forces initially increase with the solids concentration until a point at which they decrease well below the forces observed at low concentrations. This understanding was a key point in the development of an operational strategy for the system. In the graph, Test 9.1 and 9.1B have a section of pipeline in slack flow, not allowing transients to travel to the lower sections of the mine. As this slack flow section becomes smaller, the transient forces increase, until the pipeline no longer experience slack flow and the forces in the pipeline drop.

\subsection{Effect of flowrate on transients}

The effect of flowrate on the transients was also examined. For this test, the paste flowrate was gradually increased while keeping a constant paste concentration. The cylinder position and operating parameters were recorded and input into the transient model. The results were as expected; increased flowrate resulted in higher transients. It was not the magnitude of the 


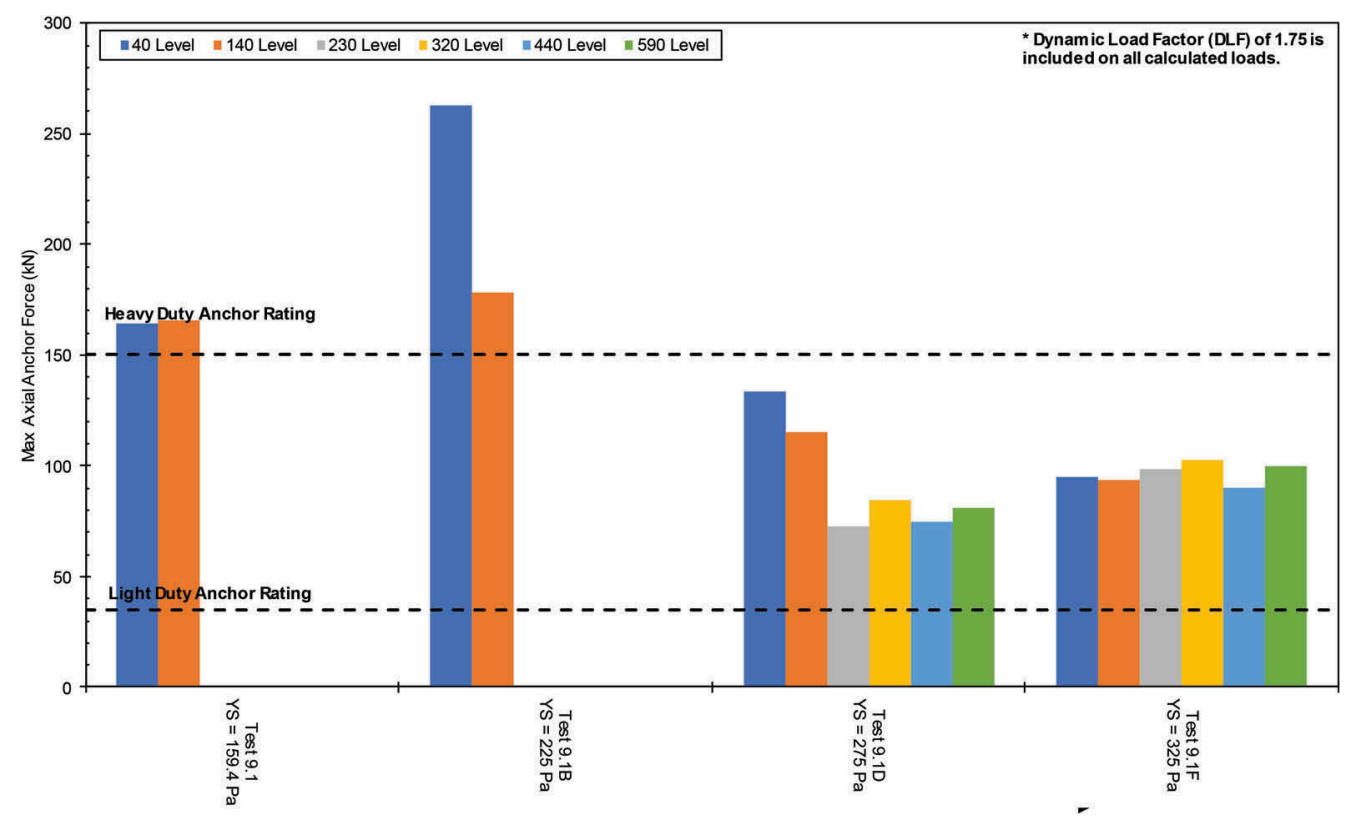

Figure 10. Effect of solids concentration on transients.

Source: Study.

flow rate as such that caused the transients but the change when the flowrate was temporarily reduced. Slower speeds results in a smaller change in flow which corresponds to a smaller transient force in the system. In the case of this specific PD pump, the cylinder position data showed that the flow was coming to a stop temporarily, irrespective of the starting flowrate.

\subsection{Effect of HPD alignment on transients}

The interaction between the HPD and the PD pump cylinder was shown to have an important effect on the transients produced in the pipeline. The goal of the HPD is to push paste into the pipeline when the cylinders are switching over in an effort to maintain a steady flowrate in the pipeline. The HPD is activated based on the position of the cylinders and its timing can be modified to achieve the best overlap. The modelling showed that if the overlap is not well adjusted, the transient forces can be greater than if the HPD was not used. This is because the flowrates of the HPD and the cylinders are added together, potentially resulting in higher flowrates than the cylinder would provide. If that flowrate then drops to zero, an even greater transient is produced than normal.

Pipeline flowrates are compared in Figure 11 to show that the effect of HPD alignment. A well aligned HPD is successful in minimising transients as shown in (a). The flowrate in this example remained mostly within $25 \%$ of the target flowrate throughout the piston transitions. This flow profile can be compared to the case where no HPD is used (b) and where the HPD is activated too early (c) or too late (d). The benefit of the HPD is evident when compared to the flow profile when the HPD is absent, where the flowrate drops to zero at every cylinder switch, resulting in large changes of flow. However, the worst case scenarios occurred when the HPD timing was misaligned. When the HPD stroke started too soon (c), the flowrate in the pipeline momentarily doubles. The early start also caused the flow to end prematurely resulting in the high flowrate to drop to zero as the next cylinder had not yet started its forward movement. The resulting change in flow is greater than that seen when the HPD was not used at all. A similar problem is seen with a late start of the HPD stroke (d). 

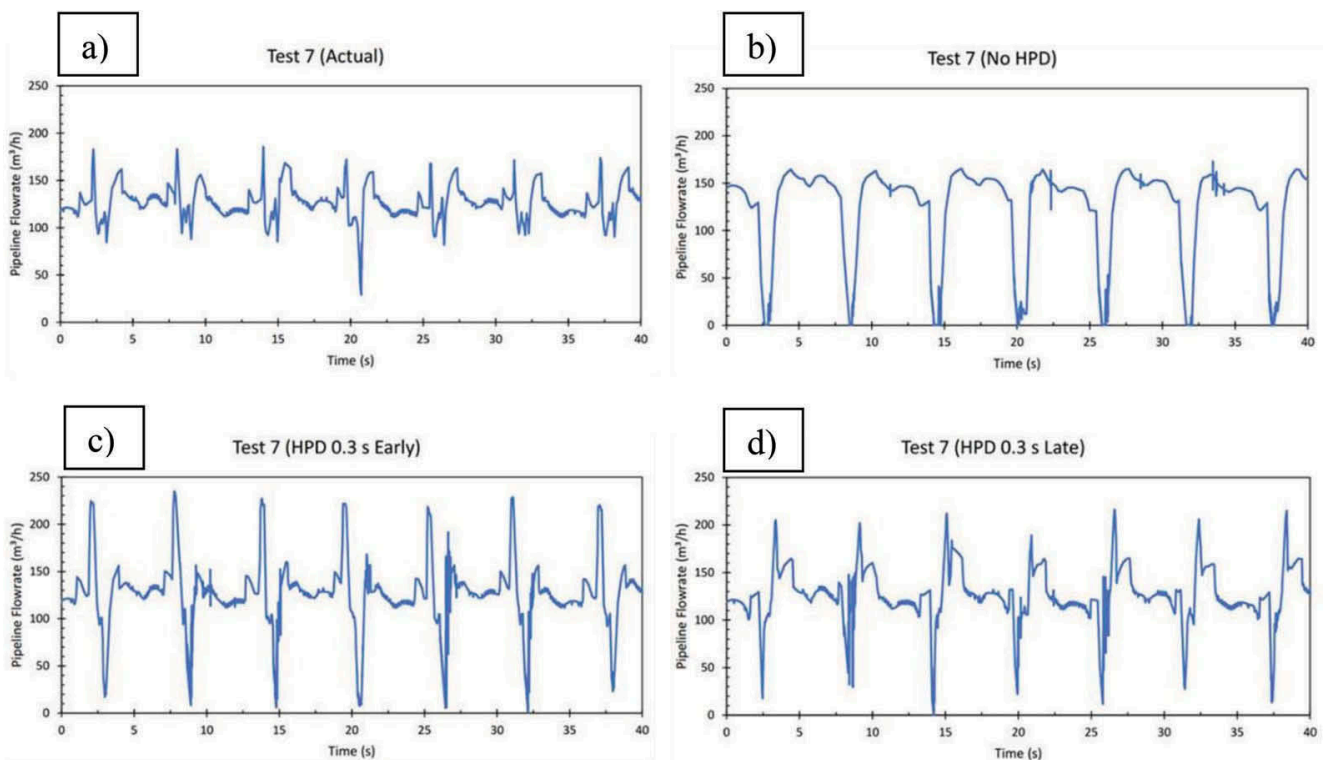

Figure 11. Flowrate profile for various HPD scenarios a) HPD aligned with PD pump strokes, b) no HPD, c) HPD starting early and d) HPD starting late.

Source: Study.

\section{OPERATING STRATEGY TO MINIMISE TRANSIENT FORCES}

\subsection{PD pump and HPD}

Since alignment between the pump and the HPD strokes were shown to have a large influence on the transients in the system, optimization of the timing is ongoing. Predictable, repeatable performance of the HPD is key to reducing the transient forces in the system and is currently under a parallel initiative on site.

\subsection{Anchor upgrades}

The anchor load capacity requirements were increased to the worst case scenario possible (misaligned HPD). Based on the transient analysis results, the heavy duty anchor (which is rated for $150 \mathrm{kN}$ ) will be used in the upper mine (level 230 and above). The normal duty anchor was upgraded to $100 \mathrm{kN}$ and will be used in the lower mine (below level 230).

Upgrades to the normal duty anchor (shown in Figure 4) included adding a cross brace between the legs and strengthening the connection point between of support legs to the pipe bracelet. Emphasis was placed on a design which could be modified in-situ on already installed anchors. Grouting of all the footings that are not currently flush to the rock is also an important part of the system upgrade, as discussed in Section 2.3.

\subsection{Process modifications}

The investigation showed that the operation methodology has a large impact on the transient forces created in the system. While the UDS system is being upgraded to the new load capacity, there are some process modification that can be taken to reduce the transient forces.

Reduce paste flow rate: A lower flowrate will reduce the overall change in flow rate during times when the HPD timing is misaligned with the PD pump strokes. Similarly, reducing the 
HPD flow rate will have a similar effect. While reducing the production rate is not a permanent solution, in the interim it will reduced the amount of downtime to repair UDS supports.

Increase solids concentration: It was clearly shown that higher solids concentration reduces the transients by putting the UDS system in a full flow condition. However, the transition to the higher solids concentration will cause higher transient forces. The strategy proposed is to start at lower concentration and lower tonnage then to increase the solids content to the desired amount. Once the system is stable and it has been confirmed that the pipeline is not in slack flow and causing large transient forces, the tonnage can then be increased. This will be a permanent operating strategy.

\section{CONCLUSION}

The investigation into the transient forces experienced in the Eleonore UDS system showed that they can be modelled based on the paste properties and the PD pump and HPD cylinder positions. A transient model was developed and subsequently validated with in-situ high speed pressure measurements. The model was used to evaluate the magnitude of the transient forces experienced in the UDS system. Under the current operating conditions, the transient forces were shown to approach and at times exceed the current load rating of the anchors.

Using the model in conjunction with field data, the effect of various process parameters on the transient forces was clarified. Key parameters included HPD timing, paste concentration and flow rate. It was found that misalignment of the HPD stroke with respect to the cylinder strokes can cause greater transients than when the PD pump is operated without a HPD at all. Temporary mitigation of transient forces can be achieved by reducing the flowrate of the pump. Increasing the solids concentration is key to maintaining a full flow pipeline, in turn lowering transient forces in the system.

Operational strategies were initiated on site to capitalize on the results of this investigation. These included upgrading the anchor support loads to match the expected transients in the system and ensuring proper installation of the support to benefit from the full load capacity of the anchors. A temporary solution is to decrease the flow rate in the system to keep the transient within the lower load capacity of the system until the proper upgrades can be completed. A permanent solution of operating with a higher paste solids concentration to ensure a full flow system has also been initiated. These changes are in addition to an ongoing effort to better align the dampener to the cylinder strokes to minimize the transient forces developed in the system.

\section{ACKNOWLEDGEMENTS}

The authors wish to express their gratitude to the Eleonore Mine for allowing the paper to be written and for providing support throughout the study.

\section{BIBLIOGRAPHY}

American Society of Mechanical Engineering 2016. ASME B31.1: Dynamic Amplification of Reaction Forces, Section II (3.5.1.3)

Study: Eleonore Pastefill UDS 2019. Completed confidentially for Newmont-Goldcorp by Paterson \& Cooke Canada Inc. 